\title{
SIMULAÇÃO NUMÉRICA DA CAMADA LIMITE PLANETÁRIA NA REGIÃO DE IPERÓ, SP-BRASIL
}

\author{
J. Scolar ${ }^{1}$, E. S. C. Neto ${ }^{1}$, A. P. de Oliveira ${ }^{2} \&$ J. Soares ${ }^{2}$
}

\begin{abstract}
A camada limite planetária (CLP) na região de Iperó-SP é estudada através de simulações numéricas utilizando um modelo unidimensional com fechamento de segunda ordem. Os resultados indicam que os fluxos verticais turbulentos, em resposta ao aquecimento solar, de calor sensível e latente têm valores máximos próximo à superfície decrescendo linearmente com a altura, favorecendo assim os transportes de calor e umidade para os níveis mais altos da atmosfera. No período noturno a turbulência térmica praticamente cessa e consequentemente os fluxos turbulentos são pequenos em toda extensão vertical da CLP. Em resposta a isto, os perfis verticais médios de temperatura e umidade são melhor simulados durante o dia. Por outro lado, os fluxos em superfície e o saldo de radiação simulados estão em quase perfeita concordância com os observados durante todo o período, e o ciclo diurno da temperatura do ar próximo à superfície é satisfatoriamente simulado.
\end{abstract}

Palavras-chave: Camada Limite Planetária; Fluxos de Calor Sensível e Latente; Temperatura na Superfície; Fluxos Turbulentos.

NUMERICAL SIMULATION OF THE PLANETARY BOUNDARY LAYER IN IPERÓ, SPBRAZIL - One-dimensional second order numerical model coupled with surface budget energy prognostic equations is used to simulate the planetary boundary layer $(P B L)$ in the region of Iperó-SP. Model results indicate that vertical turbulent sensible and latent heat fluxes decrease upwards quasi-linearly from a maximum positive value at the surface. This implies that heat and humidity are transported from the ground surface into the higher atmosphere. The mechanical turbulent flux at night is very small and the thermal flux nearly ceases in all PBL vertical extension. Consequently, temperature and humidity mean vertical profiles are better simulated during the daylight period. On the other hand, the surface fluxes and simulated radiation budget are in very good agreement with the observed values. Therefore, the inclusion of the surface energy budget equation permits a significant improvement on the air near surface daily cycle temperature simulation.

Key words: Planetary Boundary Layer; Latent and Sensible Heat Fluxes; Surface Temperature; Turbulent Fluxes.

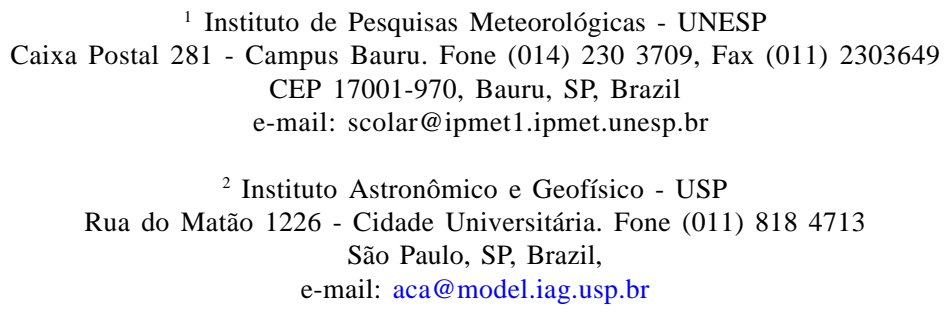




\section{INTRODUÇÃO}

A adequada simulação da camada limite planetária (CLP) é extremamente importante para a modelagem numérica da atmosfera, notadamente a de mesoescala. Inúmeros modelos numéricos têm sido desenvolvidos com o propósito de simular a camada limite planetária. Esses modelos variam desde simples parametrizações a problemas sofisticados de fechamento das equações de segunda ordem ou maiores. Contudo, exceto para o caso específico de estudos da camada limite planetária com modelo unidimensional, o tempo computacional e armazenamento em memória disponível restringem a escolha entre os vários modelos. Isso é particularmente verdadeiro para as parametrizações da camada limite planetária utilizadas em modelos de meso e grande escalas. Assim, a escolha geralmente recai sobre esquemas que utilizam parametrizações mais simples, como por exemplo os que usam o coeficiente de troca turbulenta, chamados de modelo-K. No entanto, existem certas deficiências nestes modelos, associadas, em particular, à escolha da escala de comprimento de mistura e à impossibilidade de reproduzirem o fluxos contra-gradiente. Por outro lado, os esquemas de fechamento de ordem maiores, como os esquemas de segunda ordem (Yamada \& Mellor, 1975) ou de terceira ordem (André et al., 1978), resolvem satisfatoriamente esses problemas.

Outro fator importante para a modelagem tridimensional em mesoescala é a simulação dos fluxos em superfície, principalmente a evolução e a profundidade máxima da camada limite planetária. Estas são dependentes da equipartição do saldo de radiação nos fluxos de calor latente e sensível, que por sua vez têm profunda importância na formação de nuvens nos níveis mais baixos da atmosfera (Wetzel \& Chang, 1988). Portanto, o tratamento realístico das propriedades da superfície tais como umidade do solo e características da vegetação são essenciais para simular a camada limite planetária e o desenvolvimento de cobertura de nuvens.

São encontrados na literatura vários modelos com parametrizações detalhadas da superfície do solo com a finalidade de reproduzir realisticamente os fluxos turbulentos na superfície (Deardorff, 1978; Lee \& Pielke, 1992; Mihailovic \& Kallos, 1997). Esses modelos geralmente incluem duas ou mais camadas no solo e descrevem a vegetação de modo que os fluxos turbulentos sejam fortemente dependentes da umidade do solo e do tipo de cobertura da superfície. A importância do impacto da umidade do solo na inicialização da circula- ção de mesoescala e desenvolvimento de nuvens, é mostrada nos estudos de Mahfouf et al. (1987) e Benjamin \& Carlson (1986).

O propósito deste trabalho foi investigar, com um modelo numérico unidimensional, a variação diurna da CLP na região de Iperó-SP. Os dados utilizados para inicializar o modelo foram obtidos dos experimentos de campo realizados no Centro Experimental Aramar do Ministério da Marinha $\left(23^{\circ} 25^{\prime} \mathrm{S}, 47^{\circ} 35^{\prime} \mathrm{W}\right)$ durante os períodos de 11 à 22 de março de 1991, 9 à 21 de março de 1992, 28 de julho a 7 agosto de 1992 e 8 à 21 de março de 1993. O objetivo das campanhas de medidas foi determinar a evolução temporal e espacial da CLP em regiões tropicais e subtropicais, e avaliar seu impacto na dispersão de poluentes. Os resultados parciais e descrição dos experimentos são encontrados em Oliveira (1993).

\section{DESCRIÇÃO DO MODELO}

Neste trabalho foi utilizado uma versão modificada do modelo numérico unidimensional da camada limite planetária (MCLP) para a região tropical, proposto por Oliveira (1990). Para considerar as características do solo no cálculo dos fluxos em superfície, foi incluído um modelo de duas camadas no subsolo de acordo com Deardorff (1978). Utilizou-se também o esquema de transferência radiativa desenvolvido por Katayama (1972), para o cálculo do saldo da radiação solar que atinge a superfície. Esse esquema utiliza a emissividade tabulada para o cômputo da transferência de radiação infravermelha, e uma função de absorvidade na definição de radiação solar. Inclui ainda, o efeito de cobertura de nuvens através da distribuição vertical de umidade relativa que resulta em oito configurações possíveis para nuvens, variando de céu claro a nublado com combinações de nuvens baixas, médias e altas.

O conjunto de equações unidimensional usadas para descrever os processos na CPL (Oliveira, 1990), é dado abaixo:

$$
\begin{aligned}
& \frac{\partial \bar{u}}{\partial t}=-f\left(V_{g}-\bar{v}\right)-\frac{\partial \overline{u^{\prime} w^{\prime}}}{\partial z}, \\
& \frac{\partial \bar{v}}{\partial t}=f\left(U_{g}-\bar{u}\right)-\frac{\partial \overline{v^{\prime} w^{\prime}}}{\partial z},
\end{aligned}
$$




$$
\begin{aligned}
& \frac{\partial \bar{\theta}}{\partial t}=-\frac{\partial \overline{\theta^{\prime} w^{\prime}}}{\partial z}+\frac{\partial T}{\partial t}, \\
& \frac{\partial \bar{q}}{\partial t}=-\frac{\partial \overline{q^{\prime} w^{\prime}}}{\partial z},
\end{aligned}
$$

onde,

$T=$ temperatura do ar,

$f=$ parâmetro de Coriolis,

$q=$ umidade especifica do ar,

$\theta=$ temperatura potencial do ar,

$\bar{u}=$ componente zonal do vento,

$\bar{v}=$ componente meridional do vento,

$U_{g}=$ componente zonal do vento geostrófico,

$V_{g}^{g}=$ componente meridional do vento geostrófico,

$z=$ coordenada vertical com origem na superfície da terra,

$\overline{v^{\prime} w^{\prime}}, \overline{u^{\prime} w^{\prime}}, \overline{q^{\prime} w^{\prime}}$ e $\overline{\theta^{\prime} w^{\prime}}=$ momentos estatís-

ticos de segunda ordem.

No conjunto de equações acima foram ignorados os processos de condensação e mudança de fase e os termos de advecção horizontal. Portanto, na equação da conservação de massa de vapor d'água somente o termo da divergência vertical do fluxo turbulento de umidade é considerado. Na equação da termodinâmica o balanço é obtido entre a divergência vertical do fluxo turbulento de calor sensível e o esfriamento radiativo. Os momentos estatísticos de segunda ordem são calculados através de expressões prognósticas, com fechamento de segunda ordem, onde os termos de ordem três são parametrizados seguindo a formulação proposta por Mellor \& Yamada (1982).

A taxa de variação temporal da temperatura devido à divergência do saldo do fluxo de radiação em qualquer nível de domínio do modelo é dada por:

$$
\frac{\partial T}{\partial t}=-\frac{1}{\rho c_{p}} \frac{\partial}{\partial z}\left(F_{L A}-F_{L D}\right),
$$

onde $\rho$ é a densidade do ar; $c_{p}$ é o calor específico a pressão constante; $F_{L A}$ e $F_{L D}$ são os fluxos de radiação onda longa ascendente e descendente, respectivamente, em um determinado nível de referência (Katayama, 1972). Embora existam grandes concen- trações de gases de ozônio e dióxido de carbono na atmosfera, a transferência radiativa para onda longa é limitada à absorção e emissão pelo vapor d'água. Para o cálculo de radiação de onda curta consideram-se apenas as radiações difusa e a absorvida em um determinado nível da atmosfera.

Para avaliar as condições de contorno inferior, isto é, temperatura e a umidade específica na superfície, os fluxos de calor sensível e latente são determinados através do balanço de energia na superfície com a seguinte equação:

$$
R N=H+L E+S
$$

A RN é a irradiância liquida, dada pela soma dos fluxos de radiação de onda longa e curta incidente na superfície da terra; $H$ e $L E$ são os fluxos de calor sensível e latente, respectivamente e, $S$ o fluxo de calor no solo, calculado a cada passo no tempo como resíduo dos outros fluxos. Os fluxos de calor sensível e latente são determinados através das seguintes relações:

$$
H=\rho C_{p} C_{h} U_{a}\left(\theta_{s}-\theta_{a}\right),
$$

$$
L E=\rho L C_{e} U_{a}\left(q_{s}-q_{a}\right)
$$

onde $L$ é o calor latente de evaporação; $C_{h}$ e $C_{e}$ são, respectivamente, os coeficientes de transporte de calor sensível e latente; $U_{a}, q_{a}$ e $\theta_{a}$ são a velocidade do vento, razão de mistura e temperatura potencial observados ao nível do abrigo; $q_{S}$ e $\theta_{S}$ são a umidade específica e a temperatura potencial na superfície do solo. Os coeficientes de transporte foram calculados seguindo a metodologia desenvolvida por Deardorff (1978) e $q_{s}$ é dado por:

$$
\begin{aligned}
q_{s} & =\alpha_{w} q_{s a t}\left(T_{s}\right)+\left(1-\alpha_{w}\right) q_{a}, \\
\alpha_{w} & =\frac{W_{s}}{W_{\max }},
\end{aligned}
$$

onde $q_{\text {sat }}\left(T_{S}\right)$ é a umidade específica de saturação na superfície, $T_{S}$ a temperatura da superfície, $\alpha_{\mathrm{W}}$ é a umidade relativa do solo, $W_{S}$ é a umidade da primeira camada do solo, $W_{\max }$ é a máxima umidade que o solo pode suportar. $\mathrm{O}$ fator $\alpha_{w}$ é introduzido devido ao fato que a razão de evaporação normalmente é menor do que a evaporação potencial, e pode ser especifica- 
do usando o conceito de resistência da superfície (Monteith, 1981; Kondo et al., 1990) ou ser tratado como função da umidade relativa do solo (Lee \& Pielke, 1992; Mihailovic \& Kallos, 1997; Mailhot et al., 1997). Para a obtenção da razão de mistura e da temperatura ao nível do solo seguiu-se a metodologia proposta por Deardorff (1978). Esta consiste na resolução numérica da equação prognóstica da temperatura da superfície, obtida a partir da equação do balanço da energia, considerando o solo dividido em duas camadas. Uma camada externa com espessura da ordem da profundidade do amortecimento do ciclo diurno da temperatura do solo, e uma camada interna, de espessura infinita, onde a temperatura média varia na escala anual. Assumindo que o transporte de umidade do solo ocorre de maneira análoga ao transporte de calor, Deardorff (1978) propôs a seguinte expressão para determinar a variação umidade do solo,

$$
\frac{\partial W_{s}}{\partial t}=-C_{3}\left[(E-P) / \rho_{w} D_{1}\right]-C_{4}\left[\left(W_{s}-W_{2}\right) / P_{1}\right],
$$

onde $\rho_{W}$ é a densidade da água líquida; $D_{l}$ é a profundidade da primeira camada no solo; $E$ é o fluxo de vapor d'água na superfície; $P$ é a taxa de precipitação; $W_{2}$ a umidade média da camada interna do solo, $P_{l}$ é o ciclo diurno; $C_{4}$ uma constante igual $0,90 \mathrm{e}$

$C_{3}=0,5, \alpha_{w}>0,75$

$C_{3}=14-22,5\left(\alpha_{w}-0,15\right), 0,75>\alpha_{w}>0,15$

$C_{3}=14,0,15>\alpha_{w}$.

A equação para umidade média da camada onde os fluxos turbulentos são considerados não significativos é:

$$
\frac{\partial W_{2}}{\partial t}=-\left[(E-P) / \rho_{w} D_{2}\right],
$$

$D_{2}$ é a profundidade desta camada. A equação prognóstica para temperatura do solo é,

$$
\frac{\partial T_{s}}{\partial t}=C_{1}\left(S / C_{s} D\right)-C\left[\left(T_{s}-T_{m}\right) / P_{1}\right]
$$

onde $C_{S}$ é a capacidade térmica do solo ; $D$ é a profundidade de amortecimento do solo; $S$ é o fluxo de calor no solo, $C_{l}=2 \pi^{1 / 2}, \mathrm{C}=2 \pi$ e $T_{m}$ a temperatura média diurna da segunda camada do solo.

\section{EXPERIMENTOS}

Para os testes iniciais com MCLP combinando a superfície e CLP foi utilizado o conjunto de dados observacionais obtidos no experimento micrometeorológico de campo realizado durante o período de 8 à 21 março de 1993, no Centro Experimental Aramar

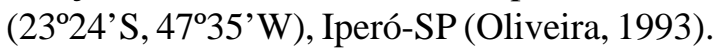

O propósito desses testes foi examinar a sensibilidade do MCLP na simulação dos fluxos de superfície e das características da CLP, com o objetivo de utilizar esses parâmetros em modelos de previsão de nevoeiro e de qualidade do ar. Os parâmetros mais relevantes calculados foram: a altura da CLP, os perfis verticais de temperatura e umidade e os fluxos turbulentos de calor sensível e latente.

Simulações de 24 horas para os dias 12 e 13 de março de 1993 foram analisadas. Estes dois dias foram selecionados por apresentarem uma ótima sequiência de dados, principalmente os coletados por balão cativo, o que permitiu visualizar a evolução temporal detalhada da camada limite planetária.

Durante os dias escolhidos o Estado de São Paulo esteve sob a influência de uma frente fria semi-estacionária com fraca intensidade no norte do Estado do Paraná, que favoreceu o surgimento de nuvens convectivas com pouca precipitação em pontos isolados.

A condição inicial do MCLP foi obtida da interpolação dos perfis de temperatura, umidade e ven-

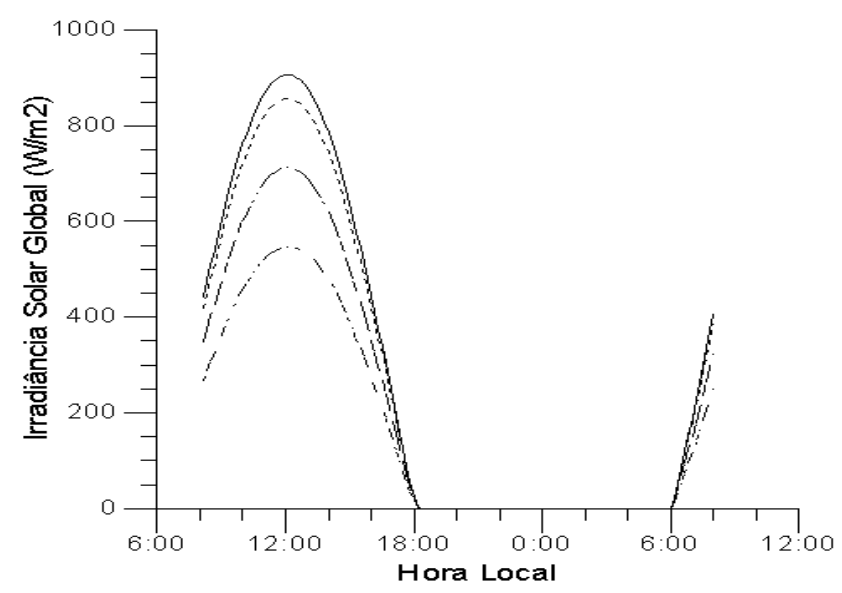

Figura 1 - Evolução temporal simulada pelo modelo da irradiância solar de onda curta, considerando: Céu claro (linha continua), somente nuvens altas (linha pontilhada), somente nuvens médias (linha tracejada) e apenas nuvens baixas ( linha tracejada- pontilhada).

Figure 1 - Model simulation of the temporal variation of the solar short-wave radiation for: Clear sky (solid line); high clouds only (dotted line): middle clouds only (dashed line) and low clouds only (dash-dotted line). 


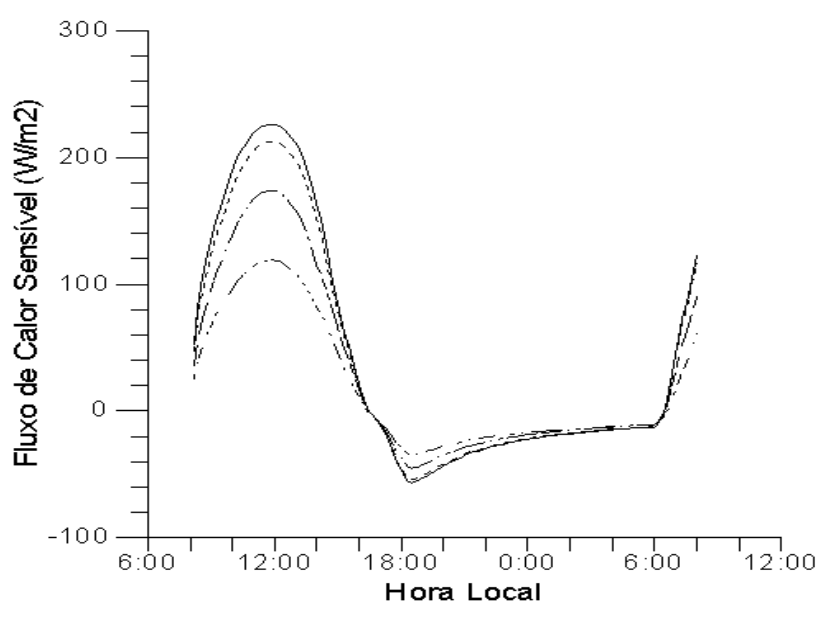

Figura 2 - Mesmo que a Fig.1, exceto para fluxo de calor sensível.

Figure 2 - As in Fig.1, except for the surface sensible heat flux.

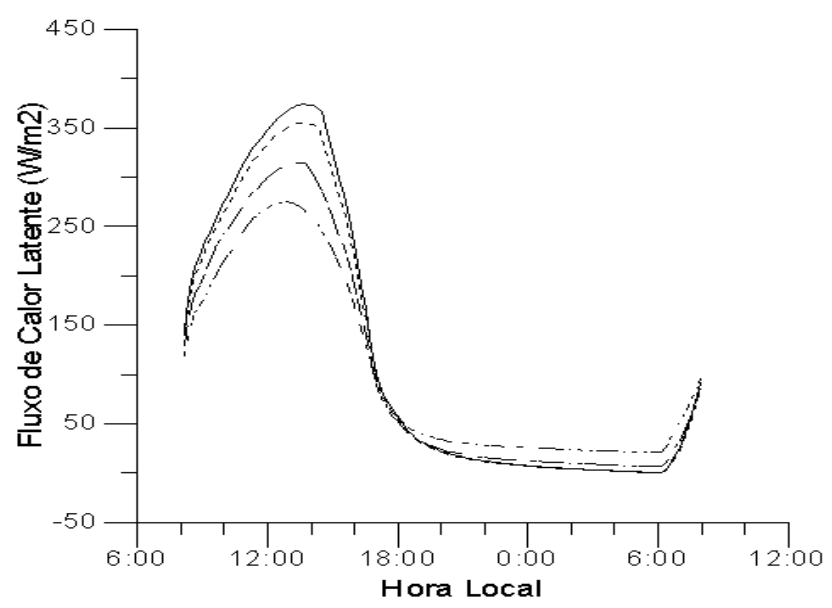

Figura 3 - Mesmo que a Fig.1, exceto para fluxo de calor latente.

Figure 3 - As in Fig.1, except for the surface latente heat flux.

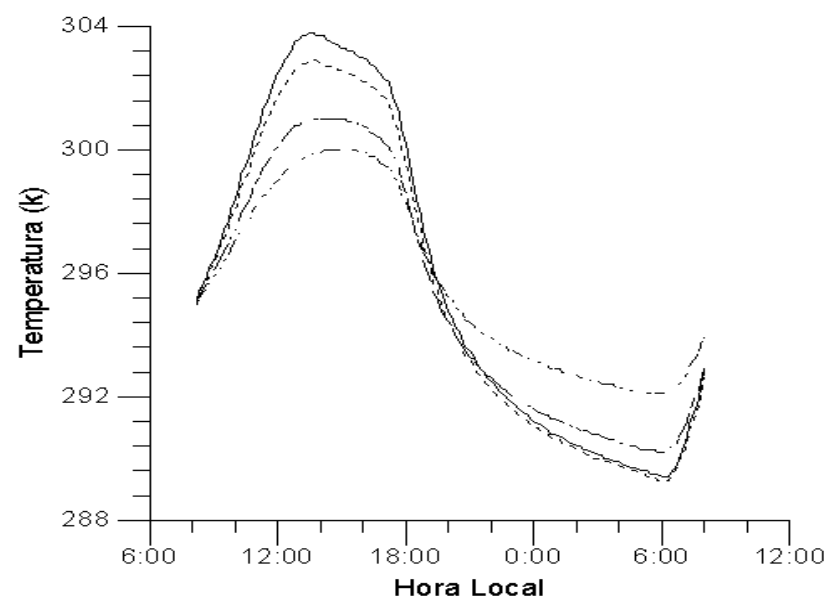

Figura 4 - Mesmo que a Fig.1, exceto para temperatura no primeiro nível do modelo.

Figure 4 - As in Fig.1, except for the temperature on the first level. tos medidos pelo balão cativo às $08: 00 \mathrm{~h}$ local (hl) do dia 12 até o nível de $890 \mathrm{mb}$. Acima deste nível e até $100 \mathrm{mb}$, os perfis iniciais foram inferidos dos dados de radiossonda localizada na cidade de São Paulo. A temperatura da camada interna do solo, foi considerada a temperatura média do dia obtida a $8 \mathrm{~cm}$ de profundidade.

Os resultados das simulações com o modelo estão mostrados nas Figs.1-4. Em cada figura quatro situações de nuvens são consideradas: sem nuvens; nuvens baixas (entre 900-750 mb), nuvens médias (entre 750-400 mb) e nuvens altas (entre 400-200 mb).

A Fig.1 mostra a radiação solar de onda curta que atinge a superfície, as Figs. 2 e 3 mostram respectivamente os fluxos de calor sensível e latente, e a Fig.4 mostra o ciclo diurno da temperatura do ar próximo à superfície.

Em todos os experimentos o saldo de radiação que atinge a superfície é equipartido basicamente entre os fluxos de calor latente e sensível. Nota-se ainda que, quanto maior a densidade de nuvens existente na atmosfera, tanto menor é a radiação que alcança o solo. Como consequiência, a temperatura do ar próximo à superfície (Fig.4) mostra o mesmo comportamento, isto é, quanto maior a radiação que atinge a superfície, maior a temperatura máxima observada. O mesmo comportamento é observado para os fluxos de calor sensível e latente (Figs.2 e 3, respectivamente), mantendo assim a consistência da equação do balanço de energia .

O fluxo de calor sensível apresenta um máximo por volta das 13 horas em concordância com o máximo da radiação de onda curta. No entanto, existe uma pequena defasagem com o máximo de calor latente, devido ao fato da primeira camada de solo apresentar

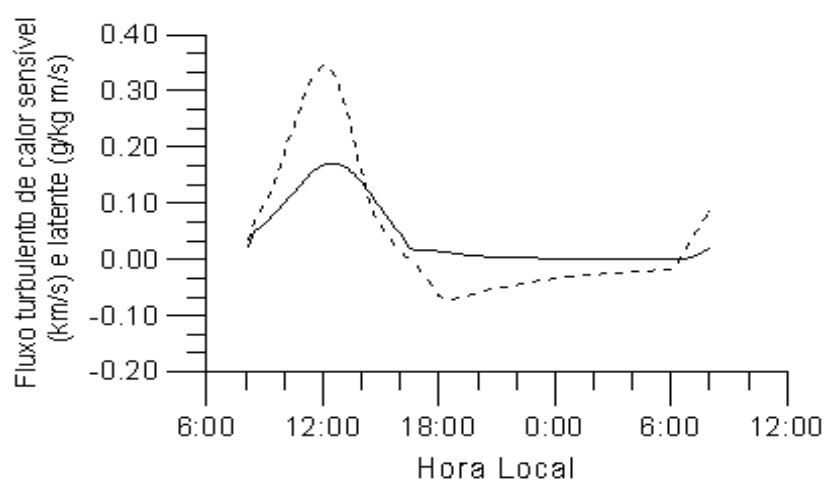

Figura 5 - Evolução temporal simulada em superfície dos fluxos turbulentos de calor sensível (linha pontilhada) e calor latente (linha continua).

Figure 5 - Simulated time variation of the surface turbulent sensible heat flux (dashed line) and surface turbulent latent heat flux (solid line). 
uma rápida secagem logo após o nascer do Sol. O valor mínimo e negativo no fluxo de calor sensível ocorre por volta das 19:00 hl. Já o fluxo de calor latente em nenhum dos experimentos apresenta valores negativos, ficando portanto em defasagem com o fluxo de calor sensível, principalmente no período noturno. Isso se deve em parte ao fato de não se ter considerado os efeitos de condensação e/ou evaporação nas equações utilizadas, e ao método do cálculo da umidade específica do solo.

A Fig.5 mostra a variação diurna dos fluxos turbulentos de calor sensível e latente na superfície. Durante o dia os fluxos crescem e alcançam valores máximos por volta das 13:00 hl, em resposta ao aquecimento solar. Obviamente, a contribuição desses fluxos decresce drasticamente a partir das 17:00 hl e permanecem assim em todo período noturno.

A evolução temporal dos perfis verticais simulados dos fluxos turbulentos de calor sensível e latente e da temperatura potencial média e umidade específica média, estão mostrados nas Figs.6-13. Durante o período diurno, os fluxos turbulentos de calor sensível (Fig.6), e de calor latente (Fig.10), são limitados pelo topo da CLP que alcança altura máxima aproximada de $1300 \mathrm{~m}$ por volta da 15:00 hl. Perfis similares são obtidos por Rogers \& Koracin (1992) e Chen \& Avissar (1994). Esses fluxos, começando com máximo valor positivo próximo à superfície, em resposta ao aquecimento solar, decrescem linearmente com a altura favorecendo o transporte de calor e umidade da superfície para os níveis mais altos da atmosfera através da

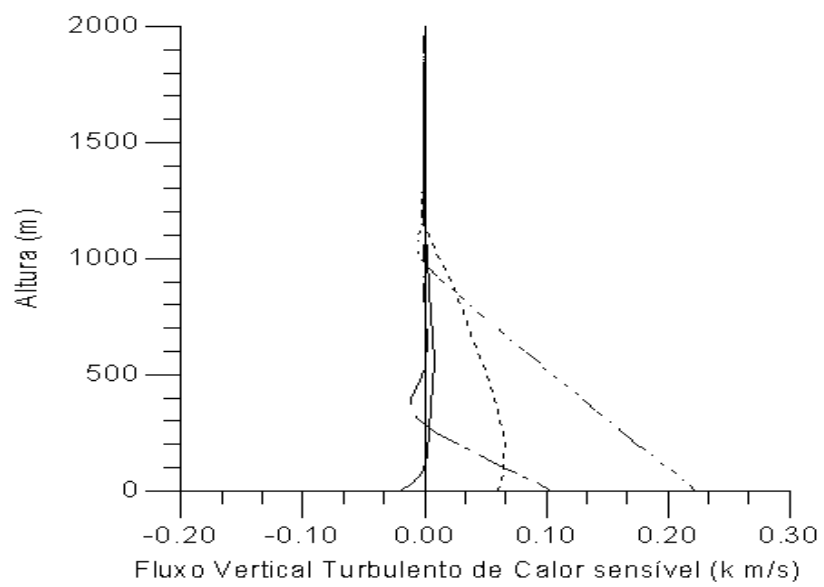

Figura 6 - Perfis vertical simulados de fluxo turbulento de calor sensível, para 09:00 hl (linha tracejada), 12:00 hl (linha tracejadapontilhada), 15:00 hl (linha pontilhada) e 18:00 hl ( linha continua).

Figure 6-Simulated vertical profiles of the turbulent sensible heat flux. The profiles are plotted at 09:00 lt (dashed line), 12:00 lt (dash-dotted line), 15:00 lt (dotted line) and 18:00 lt (solid line). circulação de pequena escala, como pode ser visto nas Figs.7 e 11, que apresentam a evolução temporal dos perfis verticais de temperatura potencial, que atinge valores de $307 \mathrm{~K}$, e da umidade específica com valores aproximados de $14 \mathrm{~g} / \mathrm{kg}$, respectivamente. Nota-se que a CLP fica bem desenvolvida ao redor das 15:00 hl, depois que os fluxos turbulentos de calor sensível e latente já atingiram os valores máximos.

Durante o período noturno os fluxos turbulentos de calor sensível e latente (Figs.8 e 12), respectivamente, são extremamente pequenos próximo à superfície e sem profundidade vertical, provocando o

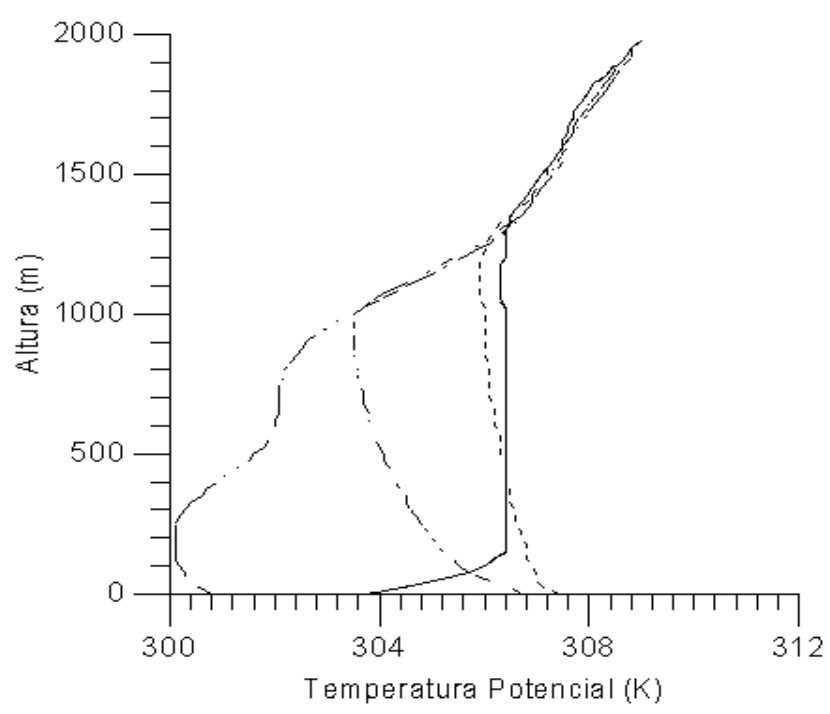

Figura 7 - Mesmo que a Fig.6, exceto para temperatura potencial.

Figure 7-As in Fig.6, except for the potential temperature.

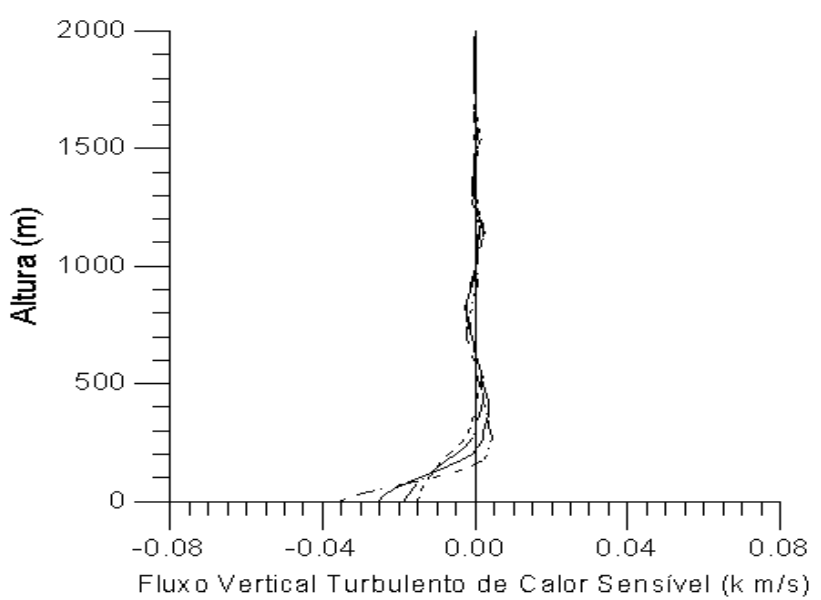

Figura 8 - Perfis vertical simulados de fluxo turbulento de calor sensível, para 21:00 hl (linha tracejada-pontilhada), 00:00 hl (linha continua), 03:00 hl (linha tracejada) e 06:00 hl (linha pontilhada).

Figure 8 - Simulated vertical profiles of the turbulent sensible heat flux. The profiles are plotted at 21:00 lt (dash-dotted line), 00:00 lt (solid line), 03:00 tl (dashed line), and 06:00 lt (dotted line). 


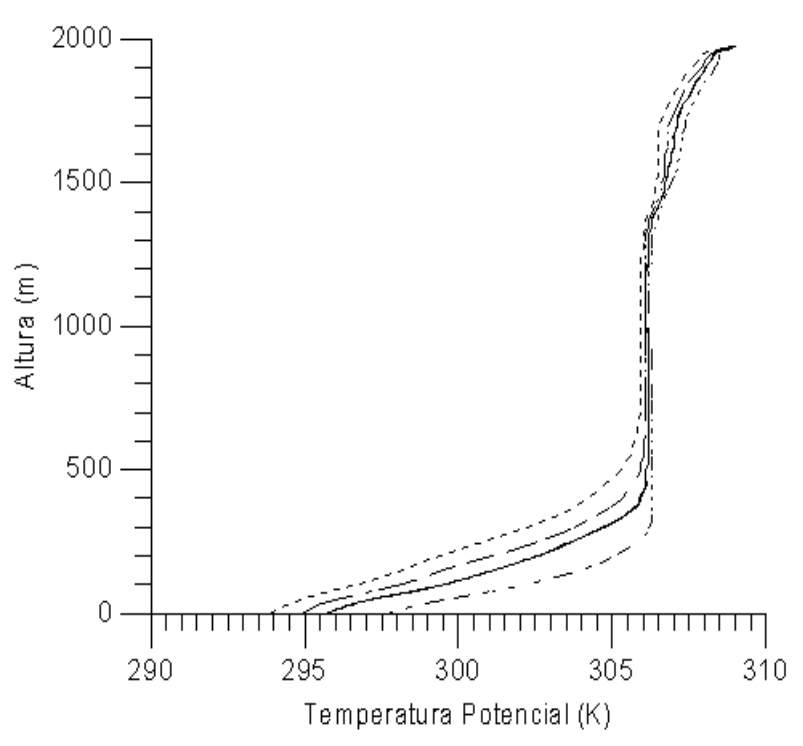

Figura 9 - Mesmo que a Fig.8, exceto para temperatura potencial.

Figure 9 - As in Fig.8, except for the potential temperature.

desacoplamento entre a camada limite estável e a camada limite planetária. Como conseqüência, as variações na temperatura potencial (Fig.9), e umidade específica (Fig.13), não são mais controladas pelo processo de turbulência térmica na CLP, mas sim pela turbulência mecânica.

\section{COMPARAÇÃO ENTRE SIMULAÇÃO E OBSERVAÇÃ̃O}

Os resultados observacionais e simulados para vários horários são apresentados nas Figs.14-18. Os fluxos de superfície, simulados do saldo de radiação, calor sensível, calor latente e de calor no solo às 08:00 hl dos dias 12 e 13 de março de 1993, são mostrados na Fig.14, enquanto que os observados estão mostrados na Fig.15.

De modo geral, os fluxos simulados apresentam o mesmo comportamento dos observados. As magnitudes dos picos diários simulados são bem reproduzidas. Entretanto, o pico no fluxo de calor latente está defasado em cerca de uma hora em relação ao saldo de radiação. É interessante notar que em ambos os casos (observação e simulação) a curva do fluxo de calor sensível cruza a curva do saldo de radiação no período da tarde, às 18:00 hl e às 07:00 hl no período da manhã, enquanto que o fluxo de calor latente cruza a curva do saldo de radiação por volta das 14:00 hl. O saldo de radiação é quase perfeitamente simulado, demonstrando que o esquema de transferência radiativa, com a formulação de condições de cobertura de nuvens, é eficiente para simulação da observação. O pico no fluxo de

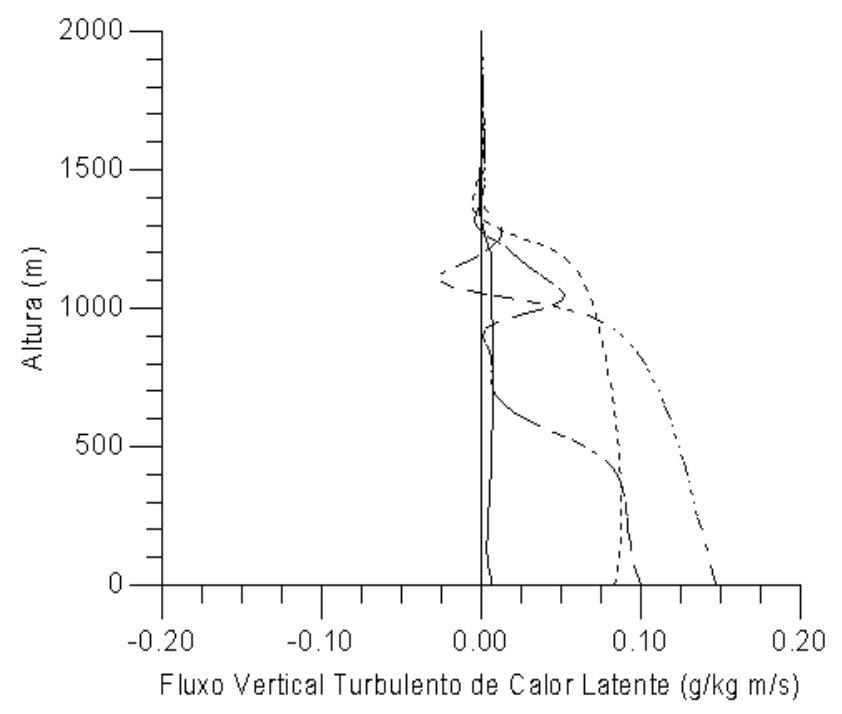

Figura 10 - Perfis vertical simulados de fluxo turbulento de calor latente, para 09:00 hl (linha tracejada), 12:00 hl (linha tracejadapontilhada), 15:00 hl (linha pontilhada) e 18:00 hl ( linha continua).

Figure 10 - Simulated vertical profiles of the turbulent latent heat flux. The profiles are plotted at 09:00 lt (dashed line), 12:00 lt (dash-dotted line), 15:00 lt (dotted line), and 18:00 lt (solid line).

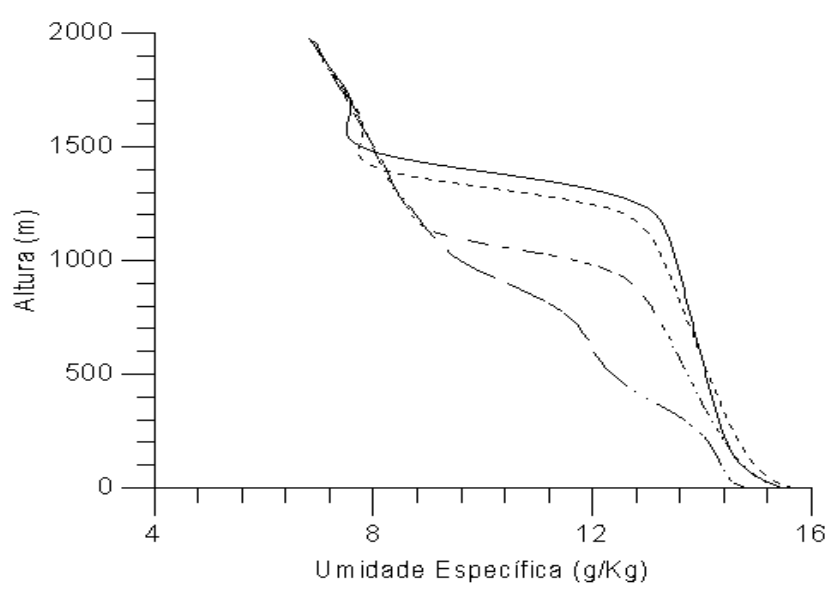

Figura 11 - Mesmo que a Fig.10, exceto para umidade específica.

Figure 11 - As in Fig.10, except for the specific humidity.

calor sensível simulado é praticamente o mesmo do observado, enquanto que o pico no fluxo de calor latente simulado é um pouco maior que o observado, o que explica a diferença encontrada no fluxo de calor no solo, que foi calculado como resíduo, na equação de balanço da energia.

A Fig.16 mostra as temperaturas, observada e simulada, ao nível de 2 metros de altura para o período das 08:00 hl do dia 12, até 08:00 hl do dia 13 de março de 1993. O MCLP reproduz claramente o ciclo diurno de temperatura. Tanto a temperatura máxima quanto a mínima estão em boa concordância com a observada. Isso se deve ao fato que no dia escolhido não houve 


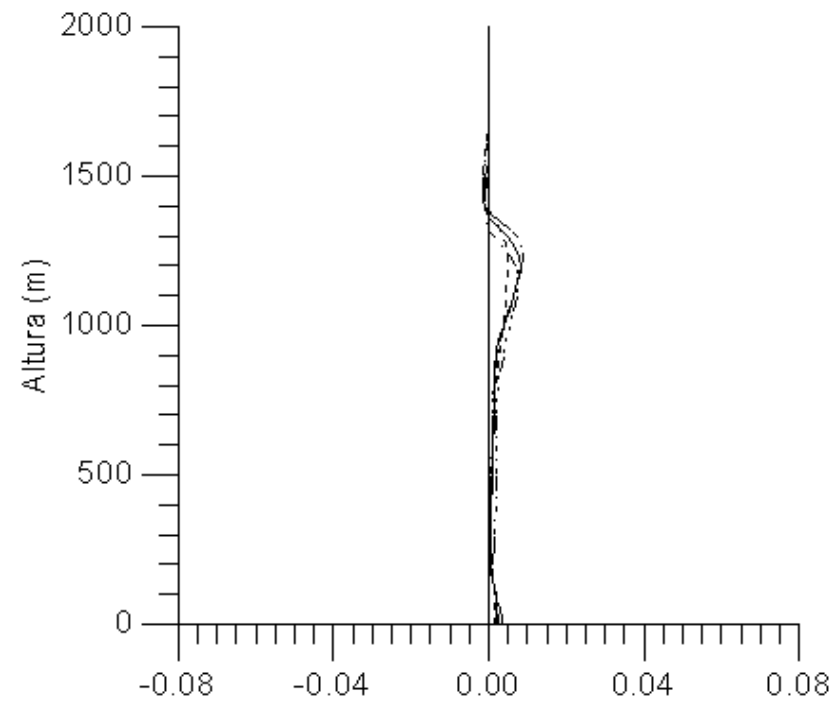

Fluxo Vertical Turbulento de Calor Latente $(\mathrm{g} / \mathrm{kg} \mathrm{m} / \mathrm{s}$ )

Figura 12 - Perfis vertical simulados de fluxo turbulento de calor latente, para 21:00 lh (linha tracejada-pontilhada), 00:00 hl (linha continua), 03:00 hl (linha tracejada) e 06:00 hl (linha pontilhada).

Figure 12 - Simulated vertical profiles of the turbulent latent heat flux. The profiles are plotted at 21:00 lt (dash-dotted line), 00:00 lt (solid line), 03:00 lt (dashed line), and 06:00 lt (dotted line).

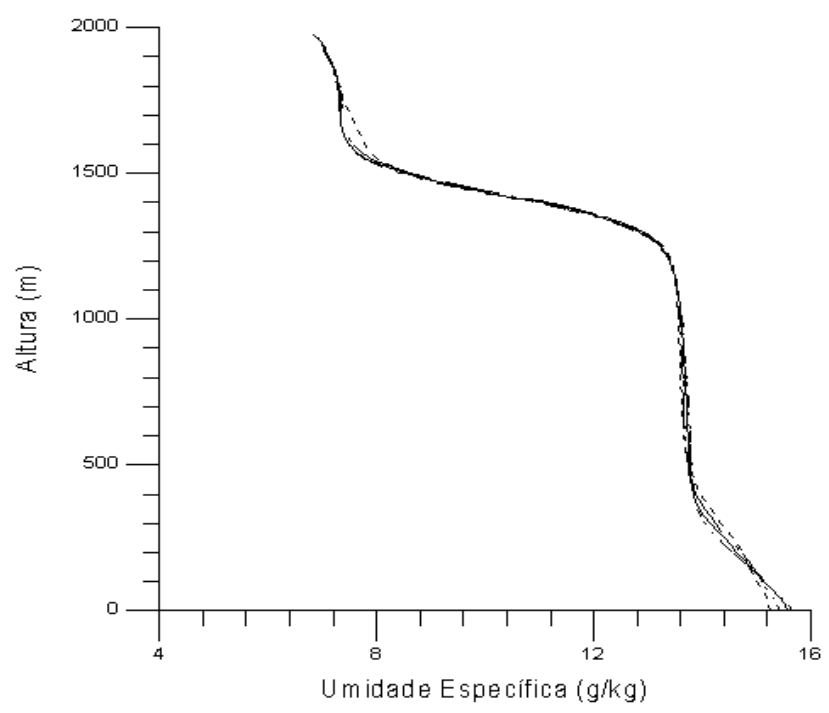

Figura 13 - Mesmo que a Fig.12, exceto para umidade específica.

Figure 13 - As in Fig.12, except for the specific humidity.

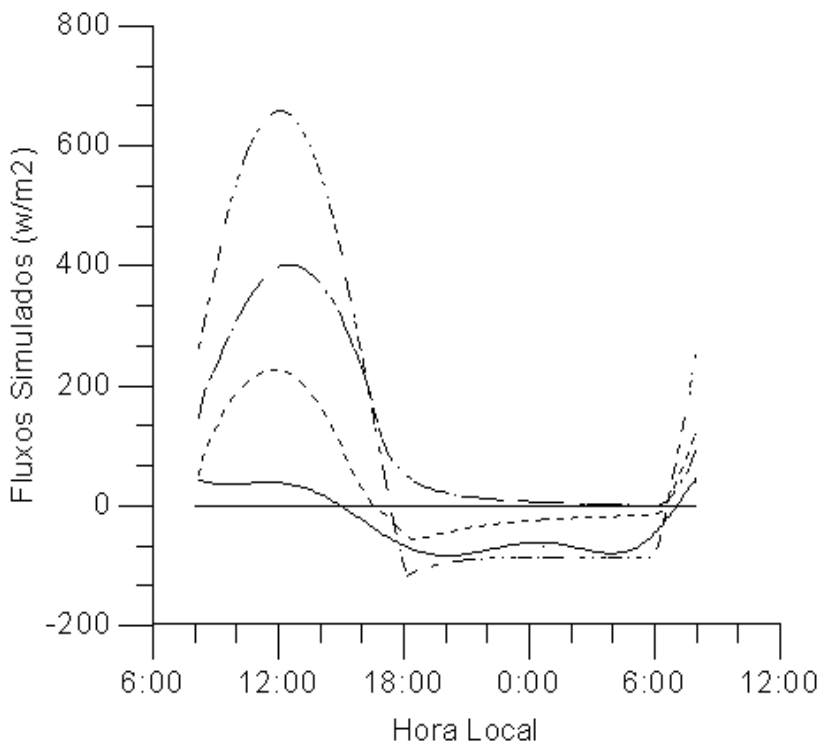

Figura 14 - Evolução temporal simulada em superfície dos fluxos de calor latente (curva tracejada), calor sensível (linha pontilhada), no solo (linha continua) e saldo de radiação (linha tracejada-pontilhada).

Figure 14 - Simulated time variation of the surface latente heat flux (dashed line), surface sensible heat flux (dotted line), surface soil heat flux (solid line), and surface net radiation (dash-dotted line).

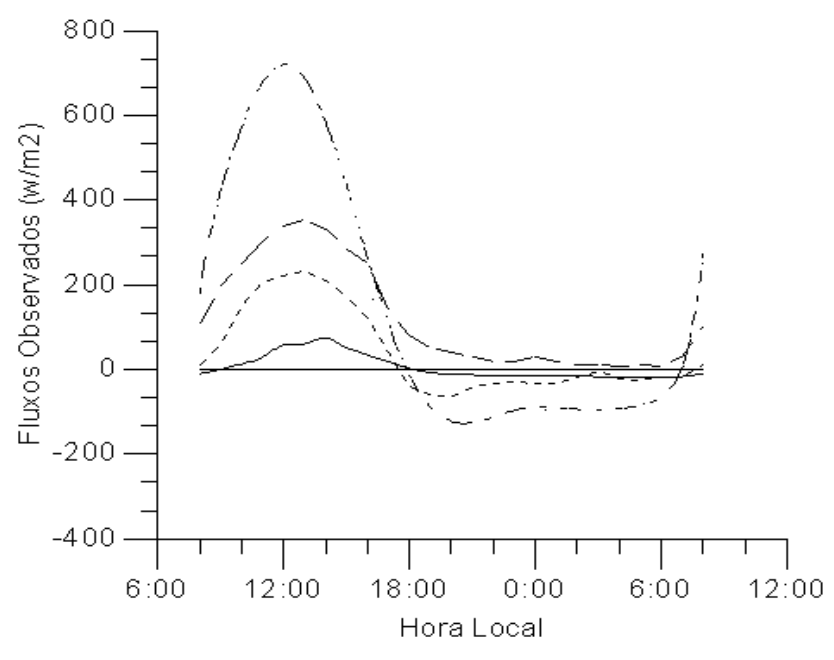

Figura 15 - Mesmo que a Fig.14, exceto para evolução temporal dos fluxos observados.

Figure 15 - As in Fig.14, except for the observed data. 


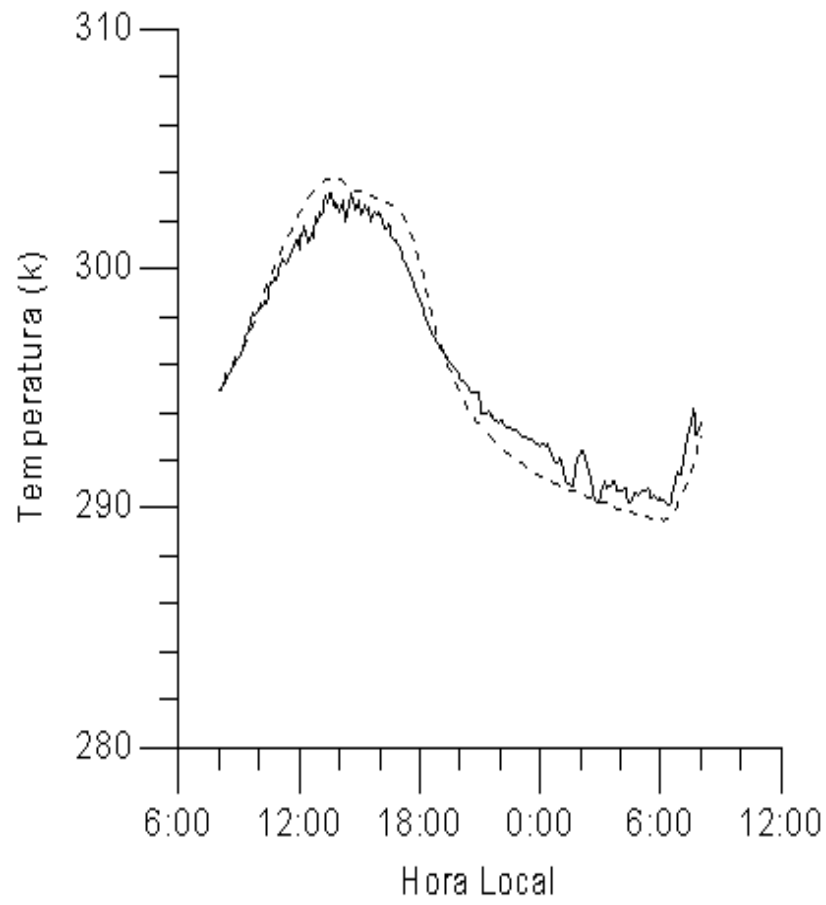

Figura 16 - Evolução temporal da temperatura observada nos dias 12 e 13 de março de 1993 (linha continua) e simulada (linha pontilhada).

Figure 16 - Time variation of the temperature observed on 12 and 13 March 1993 (solid line) and simulated (dotted line).

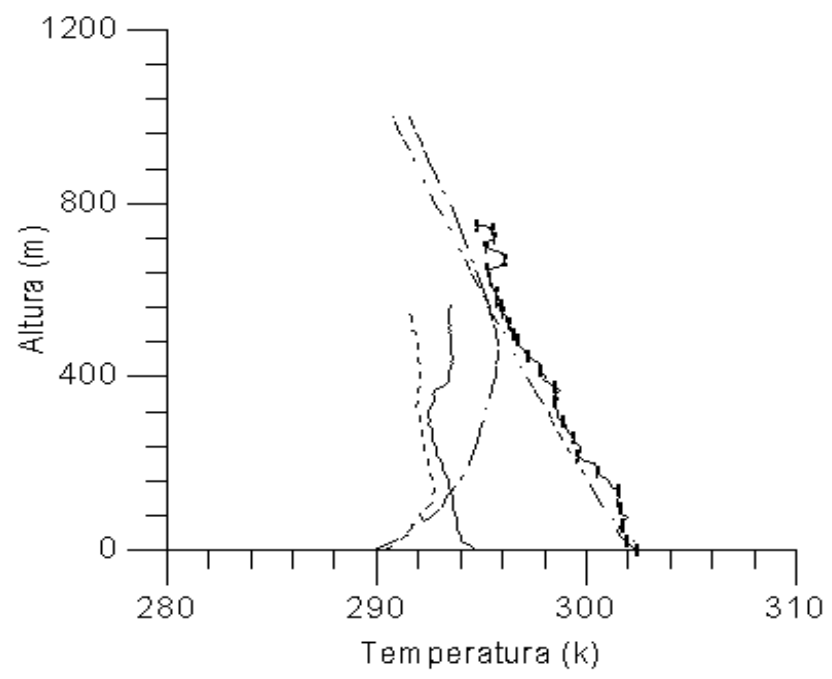

Figura 17 - Perfis vertical de temperatura observados e simulados nos dias 12 e 13 de março de 1993. Perfis observados as 08:00 hl do dia 12, usado para iniciar o modelo (linha continua), as 14:00 hl do dia 12 (circulos) e as 06:00 hl do dia 13 (linha pontilhada). Perfis simulados as 14:00 hl do dia 12 (linha tracejada-pontilhada) e 06:00 hl do dia 13 (linha tracejada).

Figure 17 - Vertical profiles of the temperature observed and simulated. Observed profiles at 08:00 lt on 12 March 1993, initial profile used for the model simulations, (solid line), 14:00 lt on 12 March 1993 (circles) and at 06:00 lt on 13 of March 1993 (dotted line). Simulated profiles at 14:00 lt on 12 of March 1993 (dashdotted line) and 06:00 lt on 13 of March 1993 (dashed line).

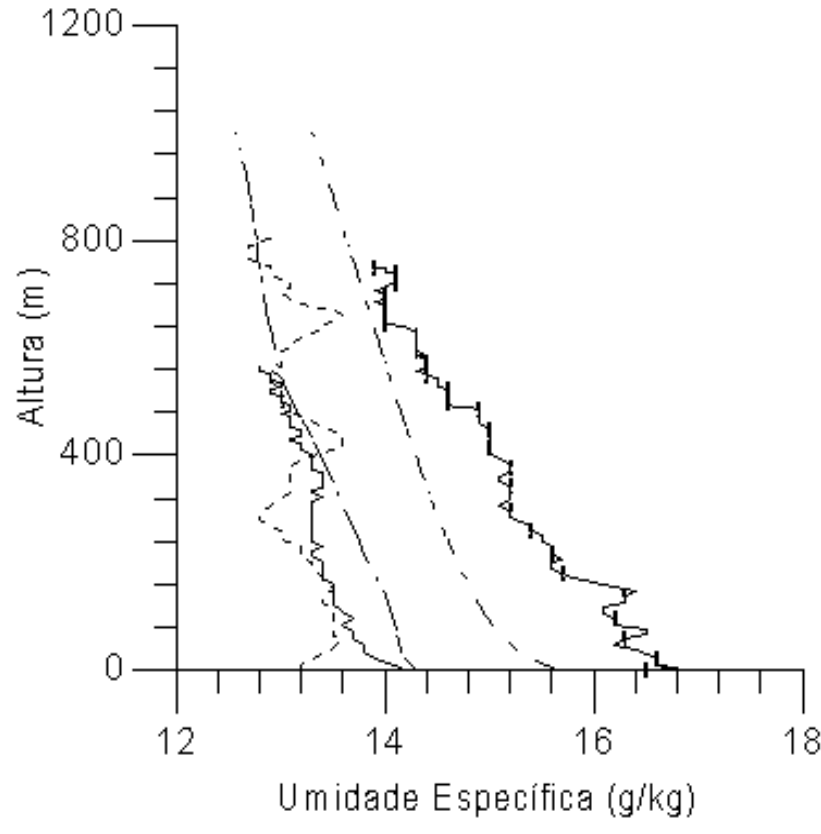

Figura 18 - Mesmo que a Fig.17, exceto para umidade específica.

Figure 18 - As in Fig.17, except for the specific humidity.

mudanças no padrão sinótico, ocorrendo pouca ou nenhuma advecção de temperatura, pouca cobertura de nuvens e sem ocorrência de precipitação.

Os perfis das temperaturas observada e calculada são apresentados na Fig.17. A curva (linha contínua fina) é o perfil observado das 08:00 hl do dia 12 (condição inicial do MCLP). Os horários escolhidos para mostrar os perfis verticais são das 14:00 hl do dia 12, próximo ao horário de temperatura máxima, e às 06:00 hl do dia 13, próximo ao horário da temperatura mínima. Durante o dia, o modelo reproduz com boa concordância o perfil de temperatura observado. A linearidade vertical que o perfil simulado apresenta indica uma camada limite bastante convectiva. No período noturno, os ventos simulados ficam extremamente calmos e conseqüentemente os fluxos turbulentos de calor sensível, latente e momentum calculados são subestimados, afetando consideravelmente a simulação dos perfis verticais das quantidades médias. A inversão de temperatura simulada é compatível com a observada, até $200 \mathrm{~m}$, que é a extensão vertical da atmosfera, onde existe fluxo vertical turbulento de calor sensível (Fig.8), embora com magnitude bem menor do que o calculado durante o dia. Acima dessa camada estável, o fluxo turbulento de calor sensível é praticamente zero e o MCLP não é capaz de reproduzir razoavelmente o perfil observado.

A Fig.18 mostra os perfis de umidade específica observados e simulados para os mesmos horários da 
Fig.17. A umidade específica é conservativa em processos isobáricos na ausência de condensação ou evaporação, na atmosfera real. Entretanto, não se encontraram essas condições ideais. Assim, durante o dia, a tendência é de aumento da razão de mistura através de evaporação próximo à superfície e de transporte de umidade para os níveis mais altos, através de convecção e transporte turbulento. Nota-se ainda na Fig.18 que mesmo no período diurno o perfil de umidade específica simulado é subestimado quando comparado com o observado. Isso é em parte devido à simplificação utilizada na equação de conservação de massa de vapor d'água, onde só os fluxos verticais turbulentos foram considerados. Durante a noite, o fluxo vertical turbulento de calor latente é praticamente nulo (Fig.12), e o modelo não consegue simular razoavelmente bem o perfil de umidade específica.

\section{CONCLUSÃO}

O objetivo deste estudo foi investigar a evolução temporal e vertical da camada limite planetária na região de Iperó-SP utilizando-se um modelo numérico unidimensional com fechamento de segunda ordem, proposto por Mellor \& Yamada (1982). Este foi acoplado ao modelo de duas camadas no solo proposto por Deardorff (1978) para determinar os fluxos de calor sensível e latente com a finalidade de determinar a temperatura em superfície utilizando a equação de balanço de energia. Na simulação, esses fluxos, bem como a variação diurna da temperatura, são consistentes com as observações. Durante o dia, a evolução temporal do perfil vertical de temperatura é bem simulado. O mesmo não ocorre durante a noite. Neste caso uma boa concordância ocorre apenas nos 200 primeiros metros. A variação temporal da umidade específica simulada tem comportamento similar à observada. Contudo, o intervalo de variação diurna simulado é menor do que o observado. Assim, os perfis simulados são subestimados durante o dia, e superestimados durante a noite. Isso é devido à simplificação utilizada nas equações, onde não se consideraram processos de evaporação e advecção horizontal.

\section{AGRADECIMENTOS}

Os autores agradecem à FAPESP pelo apoio financeiro que possibilitou a realização do experimento de campo na coleta de dados. Processo No 90/4492-1.

\section{REFERÊNCIAS}

ANDRÉ, J.C., DEMOOR, G., LACARRE, P., THERRY, G. \& DUVACHAT, R. - 1978 Modeling the 24 hour evolution of the mean and turbulent structures of the planetary boundary layer. J. Atmos. Sci., 35: 1861-1883.

BENJAMIN, S. G. \& CARLSON, T. N. - 1986 Some effects of surface heating and topography on the regional severe storm environment. Mon. Wea. Rev., 114: 307-329.

CHEN, F. \& AVISSAR, R. - 1994 - The impact of land-surface wetness heterogeneity on mesoscale heat fluxes. J. Appl. Meteor., 33: 1323- 1340.

DEARDORFF, J. W. - 1978 - Efficient prediction of ground surface temperature and moisture, with inclusion of a layer of vegetation. J. Geophys. Res., 83: 1989- 1903.

KATAYAMA, A. A. - 1972 - Simplified scheme for computing radiative transfer in the troposphere. University of California. Los Angeles, $77 \mathrm{pp}$.

KONDO, J., SAIGUSA, N. \& SATO, T. - 1990 A parameterization of evaporation from bare soil surfaces. J. Appl. Meteor., 29: 385-389.

LEE, T. J. \& PIELKE, R. A. - 1992 - Estimating the soil surface specific humidity. J. Appl. Meteor., 31: 480-484.

MAHFOUF, J. F., RICHARD, E. \& MASCART, P. - 1987 - The influence of soil and vegetation on the development of mesoscale circulations. J. Climate Appl. Meteor., 26: 1483-1494.

MAILHOT, J., SARRAZIN, R., BILODEAU, B., BRUNET, N. \& PELLERIN, G. - 1997 Development of the $35-\mathrm{km}$ version of the canadian regional forecast system. Atmosphere-Ocean, 35: $1-28$.

MELLOR, G. L. \& YAMADA, T.- 1982 Development of a turbulence closure model for geophysical fluids problems. Reviews of Geophys. and Space Physics, 20: 851-875.

MIHAILOVIC, D. T. \& KALLOS, G. - 1997 - A sensitivity study of a coupled soil-vegetation boundary-layer scheme for use in atmospheric modeling. Boundary-Layer Meteorol. 82: 283-315.

MONTEITH, J. L. - 1981 - Evaporation and surface temperature. Quart. J. R. Meteor. Soc., 77: 375401.

OLIVEIRA, A. P. - 1990 - Planetary Boundary Layer Dynamics over the Amazon Rain Forest. PhD thesis, State University of New York at Albany. New York, 296 pp. 
OLIVEIRA, A. P. -1993- Estudo da dinâmica da camada limite planetária em latitudes tropicais e subtropicais. Universidade de São Paulo- Instituto Astronômico e Geofísico. Relatório Técnico,. 70 pp.

ROGERS, D. P. \& KORACIN, D. - 1992 Radiative transfer and turbulence in the cloud topped marine atmospheric boundary layer. J. Atmos. Sci., 49: 1473- 1486.
WETZEL，P. J. \& CHANG，J.T. - 1988 Evapotranspiration from nonuniform surfaces: A first approach for short-term numerical weather prediction. Mon. Wea. Rev., 116: 600-621.

YAMADA, T. \& MELLOR, G. L. -1975- A simulation of the wangara atmospheric boundary layer data. J. Atmos. Sci., 32: 2309-2329.

\section{NUMERICAL SIMULATION OF THE PLANETARY BOUNDARY LAYER AT THE IPERÓ, SP - BRAZIL}

Numerical simulations with an one-dimensional version of a boundary layer model examined the temporal evolution of the planetary boundary layer (PBL) at the Iperó region (2325'S, 4735W'), SP-Brazil. This research is part of a project aimed to study spatial and temporal structures of PBL in tropical regions in order to assess the impact on polluent dispersion. Four fields experiments (11-22 March, 1992; 9-21 March, 1992; 28 July to 7 August, 1992 and 8-21 March, 1993) measured the mean and turbulent thermal, radiactive and aerodynamic characteristics of the surface boundary layer at the Aramar Experimental Centre meteorological tower. The $12 \mathrm{~m}$ high tower was mounted with a sonic anemometer, fine-wire thermocouple and krypton hygrometer of $10 \mathrm{~Hz}$ sample frequency at three different levels ( 3.5 and $9.5 \mathrm{~m}$ ). Temperature and relative humidity sensors were also mounted at 2.4 and $8.8 \mathrm{~m}$ heights and a Gill on propeller at the top of the tower, and net and global solar radiation, heat flux and barometer instruments at the soil surface. The PBL vertical structure was measured by tethered balloon and radiosonde, and all data collected were data logged on solid state store. The days 12 and 13 March, 1993 were selected by the high data set quality obtained by the tethered balloon in that period. Results show latent and sensible vertical turbulent fluxes having maxima values at the surface and linear decrease upwards, favouring the transportation of heat e moisture into higher atmospheric levels. At the nocturnal boundary layer, meteorological variables are not controlled by thermal turbulence and the fluxes present very small values compared with those at the daytime. Therefore, daytime temperature and humidity profiles are in much better agreement with observed ones. Similar results are obtained for latent and sensible soil fluxes and radiation budget showing the new radiative scheme and cloud cover condition implemented into the model are very efficient to simulate the surface energy budget and temperature diurnal variation near surface.

\section{NOTES ABOUT THE AUTHORS}

José Scolar

Bacharelado em Física pela Pontifícia Universidade Católica de São Paulo em 1976. Mestrado em Ciências Atmosféricas pelo Instituto Astronômico e Geofísico da Universidade de São Paulo em 1983. Atualmente é pesquisador do Instituto de Pesquisas Meteorológica (IPMet-UNESP). Suas atividades concentram-se na investigação dos processos de transporte turbulento na camada limite planetária.

\section{Ernesto S. Caetano Neto}

Bacharelado em Física pela USP em 1974, Mestrado em Física Teórica pelo Instituto de Física Teórica em 1976 e PhD. em Ciências Atmosféricas pela Reading University - Inglaterra, em 1984.

Atualmente é pesquisador do Instituto de Pesquisas Meteorológica (IPMet-UNESP). Suas atividades concentram-se na investigação Modelagem Numérica da Atmosfera, Dinâmica da Ciclogênese e de Sistemas Convectivos de Meso-escala.

\section{Amauri Pereira de Oliveira}

PhD em Ciências Atmosféricas, na State University of New York at Albany - Estados Unidos da América, em 1990, com a tese entitulada "Planetary Boundary Layer Dynamics Over the Amazon
Rain Forest". Obteve o mestrado e o bacharelado em Meteorologia no Instituto Astronômico e Geofísico da Universidade de São Paulo. Suas atividades científicas concentram-se na investigação dos processos de transporte turbulento na camada limite planetária aplicados a compreensão das circulações locais e o seu impacto na dispersão de poluentes. É professor do Departamento de Ciências Atmosféricas do Instituto Astronômico e Geofísico da Universidade de São Paulo onde leciona micrometeorologia para a graduação e pós-graduação.

\section{Jacyra Soares}

$\mathrm{PhD}$. em Oceanografia Física, pela University of Southampton, Inglaterra, em 1994, com a tese entitulada "On the reflection of equatorial waves at the eastern ocean boundaries". Obteve o bacharelado em Meteorologia no Instituto Astronômico e Geofísico e mestrado em Oceanografia Física no Instituto Oceanográfico da Universidade de São Paulo. Suas atividades de pesquisa tem se concentrado no estudo da dinâmica da grande escala oceânica e mais recentemente na área de interação oceano-atmosfera. É professora do Departamento de Ciências Atmosféricas do Instituto Astronômico e Geofísico da Universidade de São Paulo onde leciona micrometeorologia para a graduação e pós-graduação. 